NASA Technical Memorandum 101570

\title{
AEROSERVOELASTIC WIND-TUNNEL INVESTIGATIONS USING THE ACTIVE FLEXIBLE WING MODEL - STATUS AND RECENT ACCOMPLISHMENTS
}

Thomas E. Noll, Boyd Perry III, Sherwood H. Tiffany, Stanley R. Cole, Carey S. Buttrill, William M. Adams, Jr., Jacob A. Houck, S. Srinathkumar, Vivek Mukhopadhyay, Anthony S. Pototzky, Jennifer Heeg, Sandra M. McGraw, Gerald Miller, Rosemary Ryan, Michael Brosnan, James Haverty, and Martin Klepl

APRIL 1989

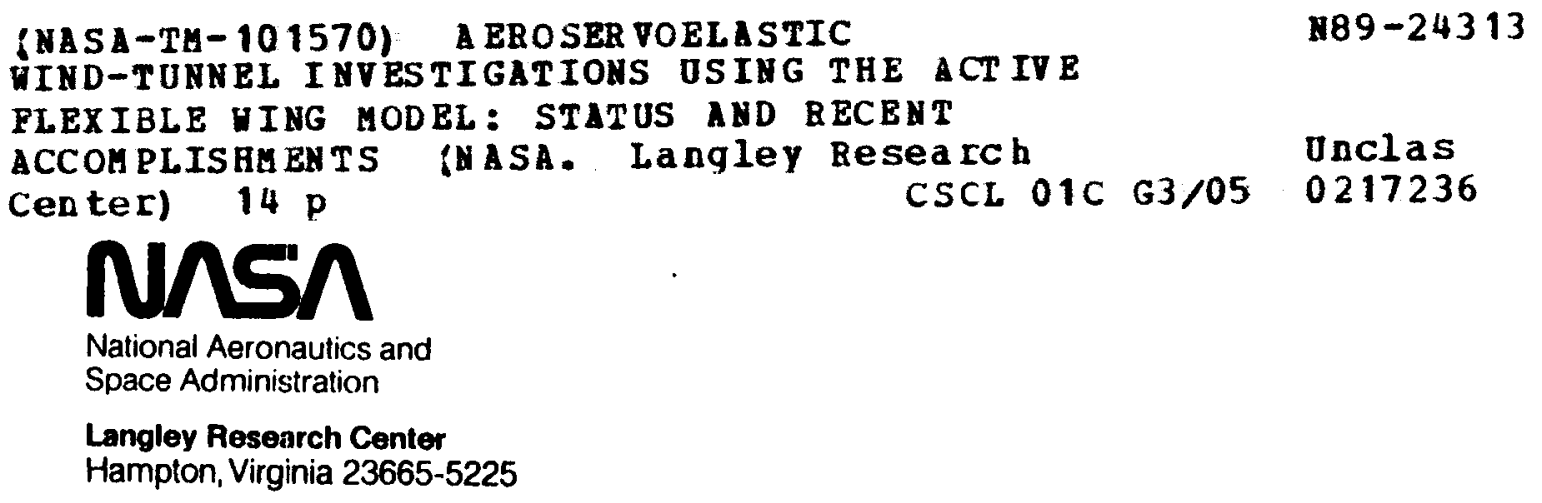




\title{
AEROSERVOELASTIC WIND-TUNNEL INVESTIGATIONS USING \\ THE ACTIVE FLEXIBLE WING MODEL - \\ STATUS AND RECENT ACCOMPLISHMENTS
}

by

\author{
Thomas Noll", Boyd Perry III, Sherwood Tiffany ${ }^{\dagger}$, Stanley Cole** \\ Carey Buttrill ${ }^{\dagger}$, William Adams, Jr. ${ }^{\dagger}$, Jacob Houck, and S. Srinathkumar \\ NASA Langley Research Center \\ Hampton, Virginia 23665 \\ Vivek Mukhopadhyay, Anthony Pototzky ${ }^{\dagger}$ Jennifer Heeg ${ }^{\dagger}$, and Sandy McGraw \\ Planning Research Corporation \\ Aerospace Technologies Division \\ Hampton, Virginia 23666 \\ and \\ Gerald Miller ${ }^{\dagger}$, Rosemary Ryan, Michael Brosnan ${ }^{\dagger}$, James Haverty and Martin Klepl ${ }^{\dagger}$ \\ Rockwell International Corporation \\ North American Aircraft \\ Los Angeles, California 90009
}

\begin{abstract}
This paper describes the status of the joint NASA/ Rockwell Active Flexible Wing Wind-Tunnel Test Program. The objectives of the program are to develop and validate the analysis, design and test methodologies required to apply multifunction active control technology for improving aircraft performance and stability. Major tasks of the program include designing digital multi-input/multi-output flutter-suppression and rolling-maneuver-load-alleviation concepts for a flexible full-span wind-tunnel model, obtaining an experimental data base for the basic model and each control concept, and providing comparisons between experimental and analytical results to validate the methodologies. This program is also providing the opportunity to improve real-time simulation techniques and to gain practical experience with digital control law implementation procedures.
\end{abstract}

\section{Introduction}

With the advent of highly flexible vehicles with multimission scenarios, active control systems that demand significant aerodynamic and structural interaction will be required. Aeroservoelasticity (ASE), a multidisciplinary technology dealing with the interactions of an aircraft's active control system and its flexible structure, has recently

$\begin{array}{ll}* & \text { Associate Fellow, AIAA } \\ * * & \text { Senior Member, AIAA } \\ \dagger & \text { Member, AIAA }\end{array}$

entered the limelight as a viable solution to questions concerning the design of a flight vehicle that meets these requirements. The ASE analysis and design methodologies that are now emerging offer the designers the capability to exploit the aircraft's aeroelastic characteristics to improve performance and stability while reducing structural weight. However, to verify the usefulness of these analysis and design methodologies it will be necessary to first perform tests and measure data on actively controlled aeroelastic wind-tunnel models and then to provide comparisons with predicted performance.

In an attempt to harness the potential benefits that may be available through the use of active controls, a research program involving the Rockwell International Corporation, the Air Force Wright Aeronautical Laboratories (AFWAL), and the NASA Langley Research Center (LaRC) was initiated in 1985 to investigate Active Flexible Wing (AFW) technology. Rockwell designed and built an actively controlled, statically and dynamically scaled, fullspan wind-tunnel model of an advanced tailless fighter. The model was tested twice (1986 and 1987) in the LaRC Transonic Dynamics Tunnel (TDT) to demonstrate, using multiple control surfaces, various concepts ${ }^{1}$ for improving aircraft roll rates.

Toextend the state-of-the-art in active controls into more challenging and rewarding areas of application, an agreement was reached in 1987 between the LaRC and Rockwell to perform cooperative AFW investigations for validating the analysis, design and test methodologies associated with multifunction digital control systems. This program provides an opportunity to design control systems, 
to improve simulation techniques, and to gain experience with digital multi-input/multi-output (MIMO) control law implementation procedures. The joint program spans approximately 3 years and will involve testing the AFW aeroelastic wind-tunnel model in the TDT.

Major tasks associated with preparing for the first tunnel entry include: 1) the derivation of the aeroelastic equations of motion using rational function approximations (RFA) of the unsteady aerodynamics and state-space techniques; 2) the synthesis of flutter suppression system (FSS) and rolling maneuver load alleviation (RMLA) control laws in the digital domain; 3) the design and development of a microprocessor digital controller and its associated hardware; 4) the simulation of the wind-tunnel model including its structural flexibility and unsteady aerodynamic effects linked to the digital controller hardware; and 5) the ground testing of the model to define its structural, dynamic and control system characteristics.

The purpose of this paper is to report on the progress and the accomplishments of the AFW Program. Although the overall program is a long way from completion, many of the tasks associated with the program are, potentially, of immediate interest to the aerospace community. The paper concentrates heavily on the analysis and design methodologies; however, to place the program in proper perspective, an overview of the entire program is provided.

\section{Wind-Tunnel Model}

The AFW Wind-Tunnel Model (Figure 1) is an aeroelastically-scaled, full-span representation of an advanced fighter configuration. The fuselage is rigid but has scaled mass and inertia characteristics. The flexible wings of the model are constructed from an aluminum honeycomb core with skins of tailored plies of a graphite/epoxy composite material oriented to permit desired amounts of bending and twisting under aerodynamic loads.

Two leading-edge and two trailing-edge control surfaces are connected to each wing semispan by hinge-linemounted, vane-type rotary actuators powered by an onboard hydraulic system. Each control surface has a chord and span of 25 percent of the local chord and 28 percent of the wing semispan, respectively. The actuators can receive constant signals from the control room or time varying signals from a computer. Deflection limits are imposed on the various control surfaces to avoid exceeding hinge-moment and wing-load limitations.

During the wind-tunnel tests, the model will be supported along the test section centerline by a sting mount. The sting utilizes an internal ballbearing arrangement with a brake device to allow the model to either roll (brake off) about the sting axis or to be held fixed (brake on) at roll angles between $\pm 90^{\circ}$. In addition, the sting has an actuator located ahead of the rolling mechanism at the model centerof-gravity for remotely pitching the model from approximately $-1.5^{\circ}$ to $+13.5^{\circ}$ angle-of-attack. Figure 2 shows the wind-tunnel model with some of the fuselage and wing panels removed to expose the internal detail. The model is instrumented with a variety of sensors that include accelerometers, strain gages, rotary variable differential transducers (RVDT), roll rate gyros, and static pressure ports.

\section{Tip Ballast}

To demonstrate FSS it was necessary to physically modify the model shown in Figure 1 so that it would flutter within the operational capabilities of the TDT. Several options were considered for lowering the flutter speed. The option most attractive was to add a ballast store on each wing tip. The tip store, shown schematically in Figure 3, is basically a thin, hollow aluminum tube with internal ballast. The addition of the tip ballast causes the wing pitch inertia and the wing total mass to increase in such a manner that the first wing bending and torsion mode frequencies are lowered and moved closer together. This causes the two modes to coalesce and cause flutter at a lower dynamic pressure than without the tip ballast present.

\section{Elutter Stopper}

The tip ballast store will also be used to provide model safety. The store is attached to the wing by a pitchpivot mechanism somewhat related to the decoupler pylon concept ${ }^{2}$ previously evaluated by NASA. The pitch-pivot mechanism uses an internal hydraulic brake mechanism such that when the brake is on for flutter testing the attachment between the wing and the ballast is essentially rigid ("stiff"); when the brake is off (either manually or automatically), a spring element internal to the store provides a more flexible ("soft") pitch stiffness: When the connection between the tip ballast and the wing is "soft," the wing first torsion mode has a higher natural frequency than when the connection is essentially rigid. Because of this higher torsional frequency, the coalescence of the wing first bending mode with torsion is delayed thereby increasing the flutter dynamic pressure. Figure 4 presents the zero-airspeed vibration characteristics for the antisymmetric first torsion mode. When the tip brake is on ("stiff"), the entire outer wing exhibits significant twisting motions; with the tip brake off ("soft"), only the tip ballast continues to exhibit large pitching motions. If the brake does not release when commanded to do so, a "structural fuse" (shear pin) has been designed to fail following large, but safe, wing deflections.

\section{Equations of Motion}

A schematic that illustrates the major analyses performed and the flow of data and information between 
analyses during the development of the equations of motion and the design of the control systems is presented in Figure 5. Circles represent both input to and output from the various analyses; rectangular boxes represent analyses. The starting point in the development of the equations of motion, at the upper left, is a circle containing a lumped-mass matrix (M) and either a stiffness $(\mathrm{K})$ or a structural-influence-coefficient (SIC) matrix from a finite element structural model tuned to match previous ground test data. This information is the input to an Eigensolver analysis that yields in-vacuum frequencies $(\omega)$, mode shapes $(\phi)$, and generalized masses $(\bar{M})$ for the first 10 symmetric and antisymmetric elastic modes. This information was obtained with the roll brake on and off and for the wing tip ballast decoupler mechanism "stiff" and "soft"

These quantities are now available for input to three other boxes, the Aeroservoelastic Analysis Tools, the Hot Bench Simulation, and the Batch Simulation. Within the Aeroservoelastic Analysis Tools box the classical open-loop aeroelastic equations of motion are generated. These equations, based on modal coordinates, are derived using Lagrange's energy equation and represent a summation of forces that include the inertial, dissipation, and internal restoring forces and the reduced-frequency dependent aerodynamic forces due to rigid-body and structural motions, control deflections, and gusts. For the antisymmetric roll brake-off configurations, a rigid-body-roll mode was appended to the aeroelastic equations. These equations are now passed "downstream" to a Control Law Synthesis Tools box and to the two simulation boxes.

When generated, control laws are passed back up to the Aeroservoelastic Analysis Tools box for computation of closed-loop frequency responses, closed-loop timeresponses, closed-loop flutter, etc. Control laws and other data are also passed to the two simulation boxes which provide a functionality check of the digital control computers and a "best" pretest estimate of the stability and performance of the closed-loop wind-tunnel model.

\section{Aerodynamic Representations}

The Doublet Lattice Lifting Surface Method ${ }^{3}$ was used to obtain the subsonic unsteady aerodynamics. For this investigation the wing tip ballast store and the fuselage were modeled as flat plates. The aerodynamic box layout for this representation is shown in Figure 6 . The location of the leading inboard (LED) and outboard (LEO) control surfaces and the trailing edge inboard (TEI) and outboard (TEO) surfaces are also denoted on the figure. Supersonic unsteady aerodynamics were generated using a modified Woodward codet.

Time-domain aeroservoelastic modeling requires rational function approximations $s^{5}$ of the reduced-frequency dependent unsteady aerodynamic force coefficients in the
Laplace domain. Several procedures for determining the RFAs are available in the ISAC ${ }^{6}$ code, the primary tool for developing the aeroelastic equations and performing ASE analyses. The techniques used during this study include the Least Squares and the Minimum-State ${ }^{7}$ Methods. Effective application of these methods requires careful study of the tabular aerodynamic data for determining and removing from consideration modes and frequency ranges that do not affect the aeroservoelastic characteristics. For the subsonic studies the Least Squares Method with optimized aerodynamic lag terms $s^{\boldsymbol{s}}$ is being used. The Minimum-State Method is used to approximate the supersonic unsteady aerodynamics.

\section{Analytical Descriotion of Actuator Drmamics}

The dynamics of the electrohydraulic actuators were modeled to best match frequency response test data. Tests indicated that the left and right actuators for each control-surface pair did not respond symmetrically. This asymmetry was taken into account by averaging the actuator responses on each side. Third-order transfer functions were obtained analytically by employing parameter estimation techniques to match the magnitude and phase characteristics of the averaged actuator frequency responses. Figure 7 shows a comparison between the experimentally-obtained frequency response and its analytical approximation for a typical control-surface pair.

\section{Control Surface Correction Factors}

To more accurately model the change of control surface effectiveness with increasing dynamic pressure, control surface correction factors derived by comparing analytical predictions with experimental data were employed. As shown in Figure 8, the leading edge surfaces generally gained effectiveness with increasing dynamic pressure, while the trailing edge surfaces lost effectiveness as shown in Figure 9. Derived correction factors varied as a function of dynamic pressure and brought the analyticallycorrected control surface stability derivatives into exact agreement with experiment.

\section{State Equations of Motion}

With the unsteady aerodynamics transformed into the Laplace domain, the equations of motion were recast into the linear time invariant, first-order equations more common for control system design applications and simulation activities. For the symmetric equations of motion based on 10 elastic modes and a single optimized aerodynamic lag term (Least Squares Method), 44 states were required (20 structural states, 10 due to the lag term, 12 due to the four thirdorder actuators and 2 for the gust mode). The total number of states changes significantly if additional lag terms are required to more accurately represent the unsteady aerodynamics. The antisymmetric equations of motion required 47 
states because of the rigidbody roll mode that was appended to the equations. For those cases where the Minimum-State Method was used, only seven aerodynamic lag states were required.

\section{Open Loop Flutter Characteristics}

The dynamic pressure root locus plot shown in Figure 10 shows some of the open loop flutter characteristics. These results are for the symmetric model and the tip ballast brake on ("stiff") at $\mathrm{M}=0.9$. Velocity was held constant and the air density was varied so that a matchedpoint solution was obtained. The predicted flutter mode involves the coalescence of the second and third elastic modes at a dynamic pressure of 213 psf and a frequency of $11.1 \mathrm{~Hz}$. The first elastic mode shown on the root locus plot is the sting bending mode. Figure 11 shows the predicted symmetric and antisymmetric flutter boundaries for this configuration with respect to Mach number.

\section{Active Flutter Suppression Design Goal and Methodology}

Mathematical descriptions of the phenomena of flutter are typically characterized by unstable, high-order models with considerable uncertainty in the model parameters. FSS designs must be robust in order to tolerate these modeling uncertainties and be able to accommodate variable test conditions (dynamic pressure and Mach number). From a practical implementation consideration the controllers must also be of low order.

\section{ESS Design Goal}

The design goal for the FSS is to increase the flutter instability boundary dynamic pressure by a factor of two. Since two separate flutter modes (a symmetric and an antisymmetric as shown in Figure 11) fall within this goal, the FSS designs must be capable of suppressing both modes simultaneously. Currently, control laws are being designed at $M=0.8,0.9$, and 1.15 by a variety of approaches explained below. Future efforts will include designs that will span the entire transonic envelope available in the tunnel using Mach/ dynamic pressure scheduling of control laws.

\section{Linear-Quadratic-Gaussian Method}

The FSS control law must be expected to satisfy a set of conflicting design requirements on performance and stability margins, yet be simple enough to be implementable on a digital microprocessor. A road map of one of the design procedures ${ }^{9}$ being evaluated to achieve this objective is provided in Figure 12. The technique first applies the Linear-Quadratic-Gaussian (LQG) Method and order-reduction techniques to develop a low-order analog control law that provides some stabilization. An optimization pro- cedure is then used to minimize a LQG-type cost function that may include dynamic loads or design RMS responses. The stability margins of the system can be improved by imposing constraints on the minimum singular value of the return difference matrix at the plant input and output. The analytical expressions for the gradients of the cost function and the constraints with respect to the control law design variables are used for this computation to facilitate rapid convergence during the optimization process. In addition, selected design responses can be incorporated as inequality constraints instead of lumping them into the cost function. This feature can be used to modify a control law to meet individual RMS response limitations and design requirements. Upon obtaining a satisfactory low-order analog controller the system is transformed into the digital domain and reoptimized, if necessary, to regain the controller performance lost due to the digitization process.

For an AFW symmetric model in which the unsteady aerodynamics were approximated using the Least Squares Method and four optimized lags (62nd-order plant), an 8th-order digital control law was obtained at a design dynamic pressure of 300 psf. A block diagram of the FSS is shown in Figure 13. The FSS, which uses the LEO and TEO control surfaces and collocated accelerometers, was able to stabilize the system up to $400 \mathrm{psf}$ dynamic pressure without gain scheduling. A minimum singular value at the plant input and output of 0.4 was also obtained at the design condition, guaranteeing MIMO stability margins of $\pm 5 \mathrm{~dB}$ in gain and $\pm 25^{\circ}$ in phase.

\section{Direct Digital, Gain Scheduled Method}

A MIMO direct digital approach is being evaluated for design at $\mathrm{M}=1.15$ using constrained optimization techniques.9-11 The approach begins with a low-order, digital control law well below the flutter boundary (perhaps 100 psf) that is designed to add damping to the wing. This starting control law may be found by a variety of methods. The LQG Method described above is currently being used. Once a satisfactory initial design is achieved the approach proceeds by finding new digital control laws at small, discrete steps in dynamic pressure (perhaps 25 psf steps) by using the constrained optimization technique. The control law from the previous step is used as the starting point in the optimization process. The design goal at each step is to add enough damping to reduce wing response to turbulence and maintain stability with the required margins. The control laws developed by this approach would require parametric scheduling in real time during the wind-tunnel tests but should provide reduced RMS loads and actuator deflections at all dynamic pressures.

\section{Stabilization Via Feedback of Synthesized Modal Velocity}

The objective of this method is to choose parame- 
ters which define linear combinations (blending) of available accelerometers so as to create sensors that observe the modal velocities of the critical flutter modes and to distribute control commands to available actuators. The goal of the controller is to prevent flutter by adding damping to the critical mode while satisfying control power, hinge moment, and other practical constraints. This design approach extends the work defined in Reference 12 by including design variables to distribute the control commands to the available control surface actuators and the incorporation of inequality constraints.

The appeal of an ideal flutter mode rate sensor can be seen by considering an open-loop transfer function consisting of a plant and the controller for the case of one critical mode being fed back. For the case where the velocity is above flutter and with a suitable selection of feedback gain, the Nyquist path is a counterclockwise circle with radius 1 centered at the -1 point (dashed curve shown in Figure 14). It is interesting to note that this is precisely the Nyquist path for the minimum energy full-state feedback Linear Quadratic Regulator (LQR) solution ${ }^{13}$. For this control law design, gain margins of -6 and $+\infty D B$ and phase margins of $\pm 60^{\circ}$ are predicted. In practice, however, one can only approximate the modal velocity sensor. FSS design analyses were performed using the two outboard and the TEI control surfaces with their collocated accelerometers and an additional tip accelerometer. This FSS provided gain margins of -6 and $+8 \mathrm{DB}$ and phase margins of $+50^{\circ}$ and $-40^{\circ}$ (solid curve shown in Figure 14) at the design point of 300 psf.

\section{Eigensystem Assignment}

When the flutter control design is formulated as a multivariable modal control problem, concepts of transmission zeros and multivariable root loci play a key role in the analysis. For this method a forward path compensator is used to assign transmission zeros to the system in order to provide adequate phase compensation at critical frequencies and also to shape the multivariable root loci. The feedback matrix is chosen based on robust eigensystem assignment theory ${ }^{14}$ using output feedback ${ }^{15}$ to stabilize the flutter mode and to optimize a performance criterion.

For this approach, the full-order model is first reduced based on the minimum Hankel norm approximation ${ }^{16}$ to obtain the "design model." Since the basic reduction algorithm is applicable only to stable systems a modal decomposition approach is used to introduce the unstable flutter mode into the "design model." Using $M=0.9$ aerodynamics the procedure was applied at a design point of 300 psf. This led to the evolution of a diagonal forward path compensator matrix with a 3rd-order compensator in each channel. Good output feedback designs were obtained with this forwar- loop compensator. A fixed-gain analog controller was subsequently digitized using the Tustin transformation and was found to stabilize the model over the dynamic pressure range from 100 to $350 \mathrm{psf}$.

Boll Plus Maneuver Load Alleviation Desion Goal and Methodology

The AFW technology approach for roll control is to twist the flexible-wing structure into an optimum shape by actively deflecting multiple leading and trailing edge control surfaces on each wing panel. A successful demonstration of this concept was performed in the LaRC TDT in 1987 using the AFW wind-tunnel model. That test demonstrated that improvements in roll rate could be achieved if the control surfaces were used to limit peak loads in addition to providing roll control.

\section{RMLA Dexien Goal}

The design goal for the RMLA system is to control wing loads at multiple points using MIMO design procedures with direct load feedback (strain-gage signals). Eight load sensors are available for feedback: midspan bending and torsion moment and wing-root bending and torsion moments on each wing panel. The design performance goals are to limit all wing loads to their maximum levels while increasing roll rate by 20 percent; or to reduce all wing loads by 20 percent for a fixed roll rate.

\section{RMLA}

The MMMO digital control law for the RMLA system will use the approach shown in the block diagram in Figure 15. Multiple wing load sensors along with a roll rate sensor will be differenced from like signals defined by the "Command Generator." This difference will be input into a feed-forward Controller which produces control surface position commands to the wind-tunnel model. The "Controller" will be designed using an LQG/LTR ${ }^{17}$ Method which will provide robust stability and good tracking of the load and roll rate commands. The load commands from the "Command Generator" will be developed for several steadystate roll rates with mathematical optimization techniques to optimally determine the minimum-load solution for a given roll rate using an analytical model of the vehicle. The optimal solution will be constrained by control surface position and hinge-moment maximums. The optimal load commands will be plotted versus roll rate command and curve fit for use in the digital controller.

\section{Digital Controller}

One of the primary objectives of the AFW Program is to gain practical experience in designing, fabricating, and implementing a real-time MIMO digital controller and in developing the hardware interface between the controller and the actual wind-tunnel model and simulator. Design specifications required that the controller have the capability 
of receiving and providing analog and discrete signals from/ to the model and the user control panel. Furthermore, it had to sample data and execute the FSS and the RMLA systems at least 200 times per second. To meet these requirements with reasonable resources, a Sun $3 / 160$ workstation driven by the Unix operating system was selected as the "shell" of the digital controller.

The hardware layout for the interface box and the digital controller is shown in Figure 16. The interface box contains the analog circuitry for processing the signals coming from or going to either the wind-tunnel model or the simulator. The circuitry includes low-pass filters (break frequencies $\geq 1000 \mathrm{~Hz}$ ) to reduce the high-frequency noise and to limit voltage spikes, antialiasing filters, and electrical isolation networks. The antialiasing filters are configured to provide either first-order roll-off with a $25-\mathrm{Hz}$ break frequency or the options of first or fourth-order roll-off with a $100-\mathrm{Hz}$ break frequency. The signals returning to the model or to the simulation computer are also filtered to prevent sharp-edge transitions from being sent to the actuators.

Besides the Sun $3 / 160$, the digital controller consists of several special purpose processors linked to the workstation via a bus. These processors include a digital signal processor (DSP), an array processor, and data translation boards. The data translation boards provide the analog-to-digital (A/D) and digital-to-analog (D/A) conversions required between the model and the controller. The DSP provides the management of all signal processing and the scheduling of the control laws. As bus master, the DSP sends the digital control commands for the actuators to the D/ $A$, sends commands to the array processor to implement the desired FSS, roll trim, and RMLA control laws, and adds digitized model excitations or bias commands to adjust camber. The DSPalso checks the user control panel switches, sets lights, and checks for faults. The array processor provides the high-speed floating-point arithmetic computations for the control laws.

The implementation of the FSS and the RMLA control laws into the control computer is a time-critical path leading up to the hot-bench simulation (HBS) and the windtunnel tests. Generic forms of the FSS and the RMLA functions were identified such that one set of software would accommodate each function while imposing minimal constraints upon the designers. The generic controller structure allows the designers to choose sensors with options to blend them, freedom of controller order with upper limits, scheduling with dynamic pressure of controller parameters, and the selection of various control surfaces with or without distribution of controller outputs. The digital controller sof tware can be modified easily and quickly as required, and the generic form of the control systems allows for changes in a design to be implemented easily and reliably.

\section{Hot-BenchSimulation}

Pretest end-to-end verification of the digital controller as a total system is essential not only for completing the goals of the program but also for model safety. For example, one of the functions to be thoroughly evaluated in the simulator is the flutter stopper. At an anticipated FSS target dynamic pressure, the open-loop flutter mode is explosive with a time-to-double amplitude of less than 60 milliseconds. The effectiveness of the tip ballast as a flutterpreventer in the "soft" configuration will be determined by clearing the test envelope in the wind tunnel. The effectiveness of the total system as a flutter-stopper, however, cannot be established in advance with the same confidence. The flutter-stopper must detect flutter transition to the "soft" configuration by activating the decoupler mechanism and damp out an established and growing oscillation.

To test the functionality of the total system the digital controller will be coupled to a HBS (Figure 17) of the model/wind-tunnel system. The ideal HBS would replicate all relevantbehavior of the model/wind-tunnel environment. As such, the HBS will include the 10 symmetric and the 10 antisymmetric flexible modes, the rigid-body roll mode, third-order actuator dynamics, and turbulence.

The AdvancedReal-Time Simulation (ARTS) System $^{18}$ at LaRC. will be used during this program. The ARTS consists of two CYBER 175 computers connected to an array of simulation sites by means of a 50-megabit-persecond fiber optic digital data network called the Computer Automated Measurement and Control (CAMAC). The CAMAC interface converts CYBER 175 digital signals to analog signals which are sent to the AFW control computer through the wind-tunnel interface unit. In addition the CAMAC site converts analog signals coming from the AFW control computer to digital signals to be sent to the CYBER 175. The various simulator sites include real-time control consoles, engineer's consoles, aircraft cockpits, graphics computers, minicomputers, etc. For this program an Adage Graphics Computer, which is interfaced directly to the CYBER 175, will be used to display a color-coded, threedimensional wireframe outline of the AFW Model. The display presents aircraft pitch, roll and yaw angles, control surface deflections, and total model deformation.

The size of the HBS model and its highest frequency dynamics prevent it from being run in actual real time on the Cyber computers. An integration time step of 1/ 2000 seconds is anticipated. As a result, the HBS will be coupled to the digital controller in synchronized "slowtime" with a time-scale factor of 20:1. The HBS will use the normal integration step size of $1 / 2000$ seconds but will update at a sample time of $1 / 100$ seconds. The digital controller will run exactly. as it would in the wind tunnel except that for the HBS test it will sample every $1 / 10$ of a second instead of every $1 / 200$ of a second. To maintain 
dynamic fidelity a separate set of antialiasing filters which have break frequencies at $5 \mathrm{~Hz}$ are available for the model controller interface box for use with the HBS. In addition, the digital controller outputs will be sent to the D/A at the beginning of a computational frame to insure that almost a complete frame exists between the inputs and outputs of the digital controller no matter whether it is running with the HBS or with the actual model.

An independent batch simulation has also been developed to support the control law designs and analyses and to provide an independent check for the HBS (see Figure 5). The batch simulation is coded in Advanced Continuous Simulation Language (ACSL) ${ }^{19}$ and has been verified by comparing ACSL-generated and ISAC-generated linear analyses.

\section{Test Plans}

\section{Ground Tests}

The intent of ground testing is to obtain as much measured data as possible for validating the math models at zero-airspeed and to verify the model's structural integrity. The goal of the planned ground vibration test (GVT) is to measure the vibration frequency, mode shape, damping, and generalized mass for each of the 10 symmetric and 10 antisymmetric modes. These measurements will be made for both the "stiff" and "soft" configurations of the wing-tip ballast. The majority of the GVT measurements will be made with sine-dwell techniques using a multiple-electromagnetic shaker arrangement. Some measurements will be made using a calibrated hammer as a supplement to the sinedwell information. During these tests all actuators will be hydraulically powered. While these are the goals of the GVT it is noted that physical measurements of both structural damping and generalized mass are quite difficult and that reasonable results may not be attainable.

Open-loop end-to-end tests will be accomplished to obtain transfer functions over a broad frequency range for all control surface/sensor combinations using several different amplitude signals to evaluate the nonlinear effects. The transfer functions of the actuators will also be measured in an unloaded condition.

\section{Wind-Tunnel Tests}

The goal of the first wind-tunnel entry scheduled for the summer of 1989 is to measure flexibilized stability derivatives to define the unaugmented aeroelastic characteristics for "stiff" and "soft" tip ballast configurations and to demonstrate RMLA and FSS control laws. All testing will be accomplished in an order of increasing risk to the model. The goal of the second test entry scheduled 1 year later is to investigate multifunction digital control law design capabil- ity by demonstrating FSS and RMLA systems, simultaneously.

The capability to provide near real-time controller performance evaluation (rapidly assess the robustness characteristics of MIMO control laws) to assist in the decision on whether to continue testing is currently being reviewed. To be feasible, the capability must include: 1) the transfer matrix estimation of the closed-loop system;2) the computation of the open-loop transfer matrix knowing the measured closed-loop transfer function and the programmed control law; 3) the formation of matrices required for singular value and/or eigenvalue computations; and 4) the graphical display of results with hardcopy capability. All of the above tasks need to be accomplished in just a few minutes to be practical.

\section{Concluding Remarks}

This paper has addressed the questions of why the AFW Program is being pursued, where we are today, and what is planned for the next several years. Hopefully, the paper has also provided an early transition of this developing technology to the aerospace community. Some of the more significant accomplishments are described below.

1) A wing-tip ballast was designed and fabricated for the AFW Model to create a flutter condition within the TDT for FSS demonstrations.

2) The structural dynamic effects of the wing-tip ballast can be quickly altered during the tests using a decoupler pylon mechanism for passively preventing flutter.

3) The use of rational function approximations of unsteady aerodynamics with improved options of applying physical weighting, constraints, and optimization provides smaller-order matrices for the control system design and simulation activities.

4) Several advanced design approaches have been used to obtain preliminary FSS control laws capable of significantly increasing the flutter dynamic pressure and providing realistic gain and phase margins.

5) A digital controller consisting of a Sun $3 / 160$ with a separate digital signal processing board and an array processing board was designed and fabricated. 


\section{References}

1. Perry, B., III; Dunn, H. J.; and Sandford, M. C.: Control Law Parameterization for an Aeroelastic Wind-Tunnel Model Equipped with an Active Roll Control System and Comparison with Experiment. AIAA Paper No. 88-2211, presented at the AIAA 29th Structures, Structural Dynamics and Materials Conference, April 1988.

2. Reed, W. H., III; Foughner, J. T. , Jr.; and Runyan, H. L., Jr.: Decoupler Pylon: A Simple, Effective Wing/Store Flutter Suppressor. AIAA Journal of Aircraft, Vol. 17, No. 3, pp. 206-211, March 1980.

3. Geising, J. P.; Kalman, T. P.; and Rodden, W. P.: Subsonic Unsteady Aerodynamics for General Configurations, Part I: Direct Application of the Nonplaner Doublet Lattice Method. AFFDLTR-71-5, 1971.

4. Clever, W.: Subsonic/Supersonic Linear Unsteady Analysis. AIAA Paper No. 85-4059, presented at the AIAA 3rd Applied Aerodynamics Conference, October 1985.

5. Tiffany, S. H.; and Adams, W. M., Jr.: Nonlinear Programming Extensions to Rational Function Approximations of Unsteady Aerodynamics. AIAA Paper No. 87-0854, presented at the AIAA 28th Structures, Structural Dynamics and Materials Conference, April 1987.

6. Peele, E. L.; and Adams, W. M., Jr.: A Digital Program for Calculating the Interaction Between Flexible Structures, Unsteady Aerodynamics, and Active Controls. NASA TM-80040, 1979.

7. Karpel, M.: Design for Active and Passive Flutter Suppression and Gust Alleviation. NASA CR-3482, November 1981.

8. Tiffany, S. H.; and Karpel, M.: Aeroservoelastic Modeling and Applications Using Minimum-State Approximations of Unsteady Aerodynamics. AIAA paper presented at the AIAA 30th Structures, Structural Dynamics and Materials Conference, Mobile, Alabama, April 1989.

9. Mukhopadhyay, V.: Digital Robust Active Control Law Synthesis Using Constrained Optimization. AIAA Journal of Guidance, Control and Dynamics, Vol. 12, No.2, March-April 1989.
10. Mukhopadhyay, V.; Newsom, J. R.; and Abel, I.: A Method for Obtaining Reduced Order Control Laws for High Order Systems Using Optimization Techniques. NASA TP-1876, August 1981.

11. Mukhopadhyay, V.: Digital Active Control Law Synthesis for Aeroservoelastic Systems. Presented at the 1988 American Control Conference, June 1988.

12. Johnson, E. H.; Hwang, C.; Joshi, D. S.; Kesler, D. F.; and Harvey, C. A.: Test Demonstration of Digital Control of Wing/Store Flutter. AIAA Journal of Guidance, Control and Dynamics, Vol. 6, No. 3, pp 176-181, May-June 1983.

13. Adams, W. M., Jr.; Fennell, R. E.; and Christhilf, D. M.: Flutter Suppression Using Eigenspace Freedoms to Meet Requirements. Paper Presented at the 2nd NASA/Air Force Symposium on Recent Advances in Multidisciplinary Analysis and Optimization, September 1988.

14. Srinathkumar, S.: Robust Eigenvalue/Eigenvector Assignment in Linear State Feedback Systems. Proceedings from the 27th IEEE Conference on Decision and Control, pp 1303-1307, 1988.

15. Srinathkumar, S.: Eigenvalue/Eigenvector Assignment Using Output Feedback. IEEE Transactions Automatic Control, Vol. AC-23, No. 1, pp 79-81, 1978.

16. Glover, K.: All Optimal Hankel-Norm Approximations of Linear Multivariable Systems and Their K. Error Bounds. International Journal of Control, Vol.39, No. 6, pp 1115-1193, 1984.

17. Ridgely, D. B.; and Nanda, S. S.: Introduction to Robust Multivariable Control. AFWAL-TR-853102, February 1986.

18. Crawford, D. J.; Cleveland, J. I., II; and Staib, Richard O.: The Langley Advanced Real-Time Simulation (ARTS) System Status Report. AIAA88-4595-CP, September 1988.

19. Advanced Continuous Simulation Language (ACSL) Reference Manual, Fourth Edition. Mitchell and Gauthier Associates, Concord, MA, 1986. 


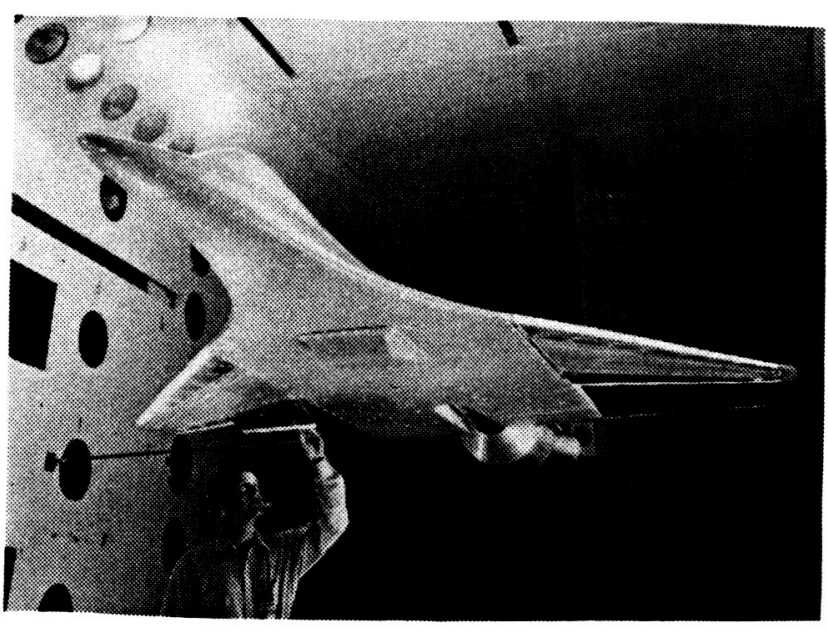

Figure 1 AFW model mounted in the NASA LaRC TDT



Figure 2 Internal details of the wind tunnel model

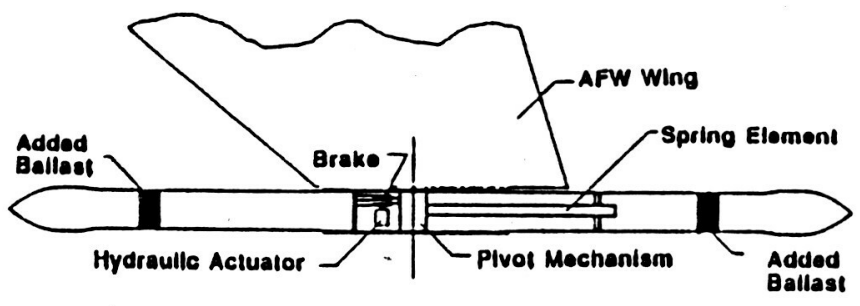

Figure 3 Tip ballast store for the wind tunnel model

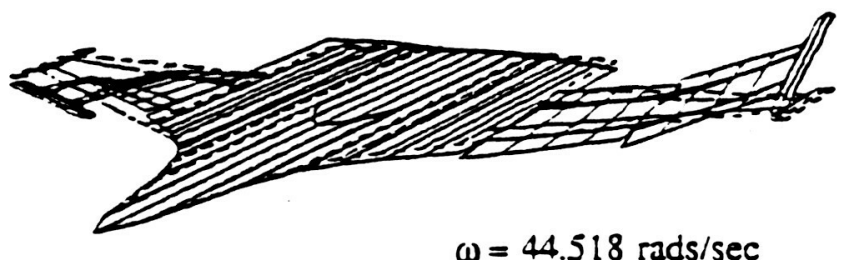

$\omega=44.518 \mathrm{rads} / \mathrm{sec}$

"STIFF" CONFIGURATION

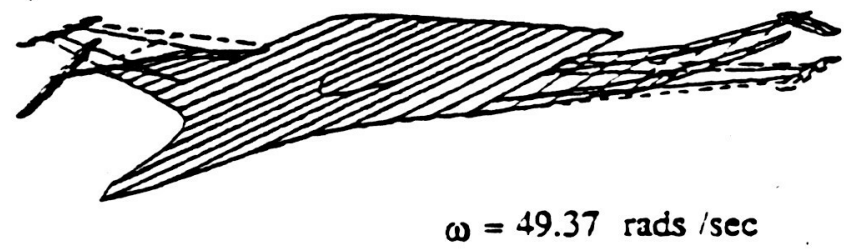

"SOFT" CONFIGURATION

Figure 4 Antisymmetric torsion mode shapes for the tip ballast "Stiff" and "Soft"

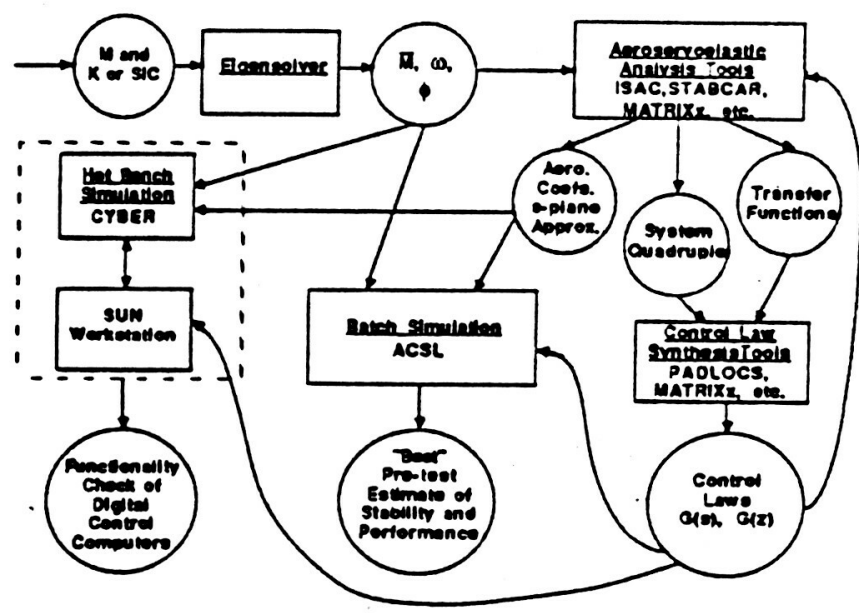

Figure 5 Schematic of the analysis procedure 


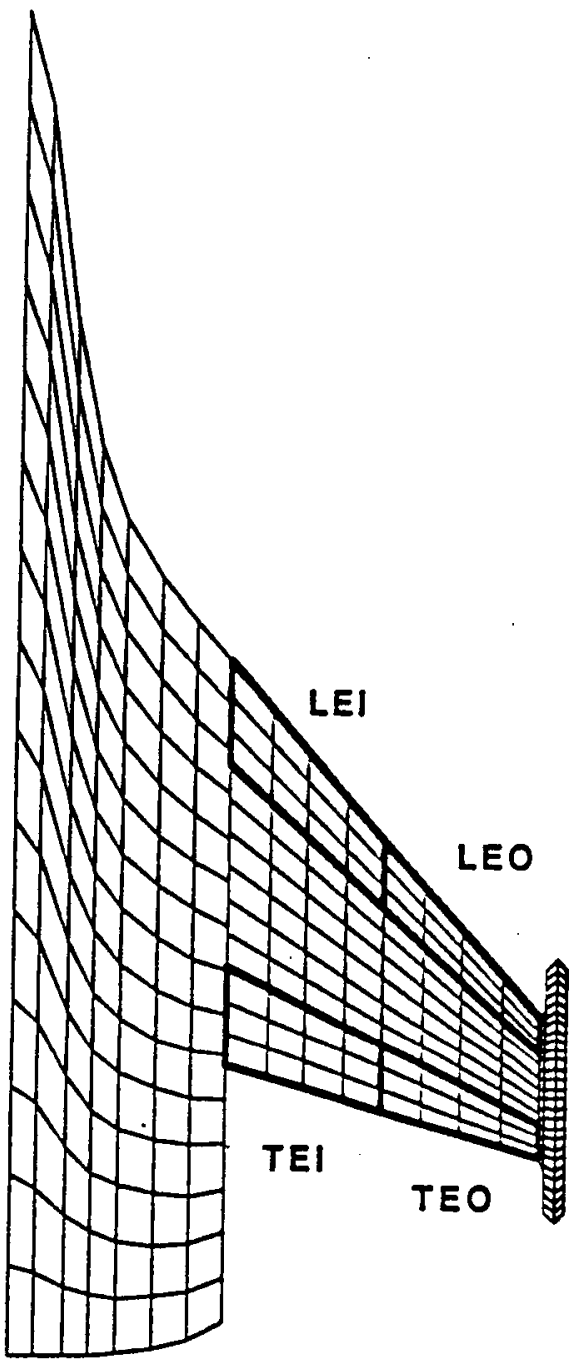

Figure 6 Aerodynamic representation of the model for subsonic analyses

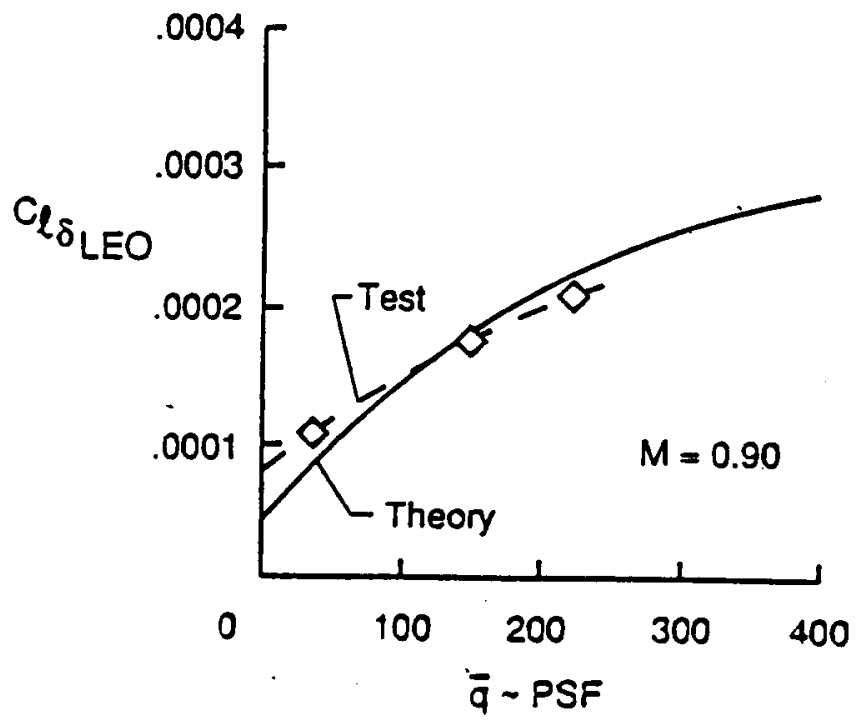

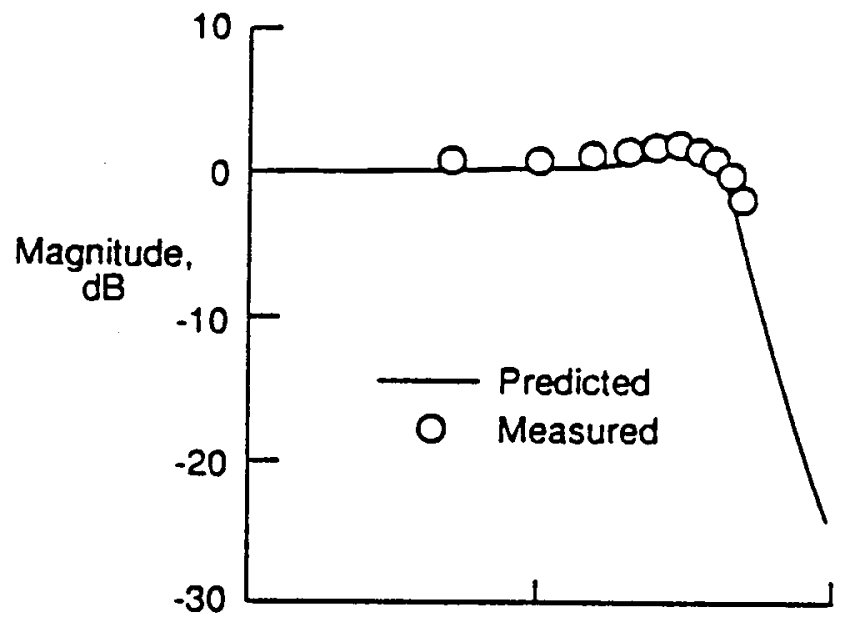

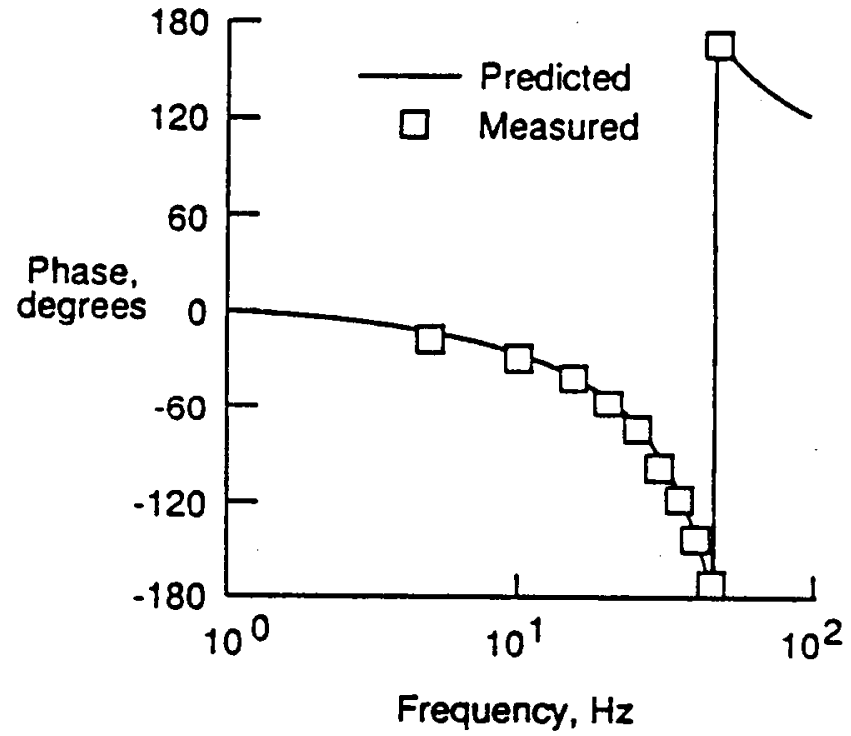

Figure 7 Comparisons between predicted and measured actuator transfer functions

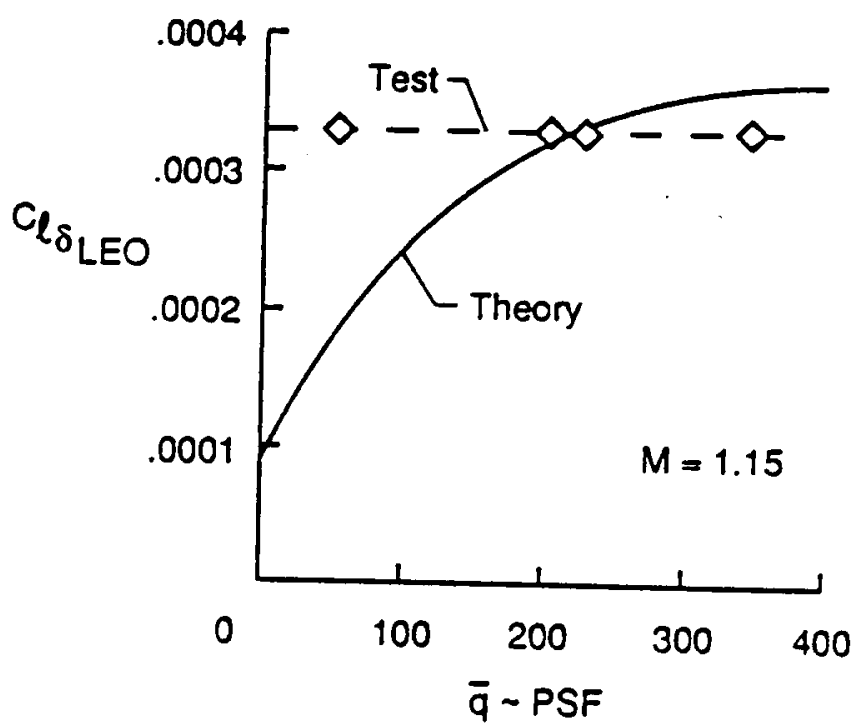

Figure 8 Leading edge outboard control surface roll effectiveness with Mach number 


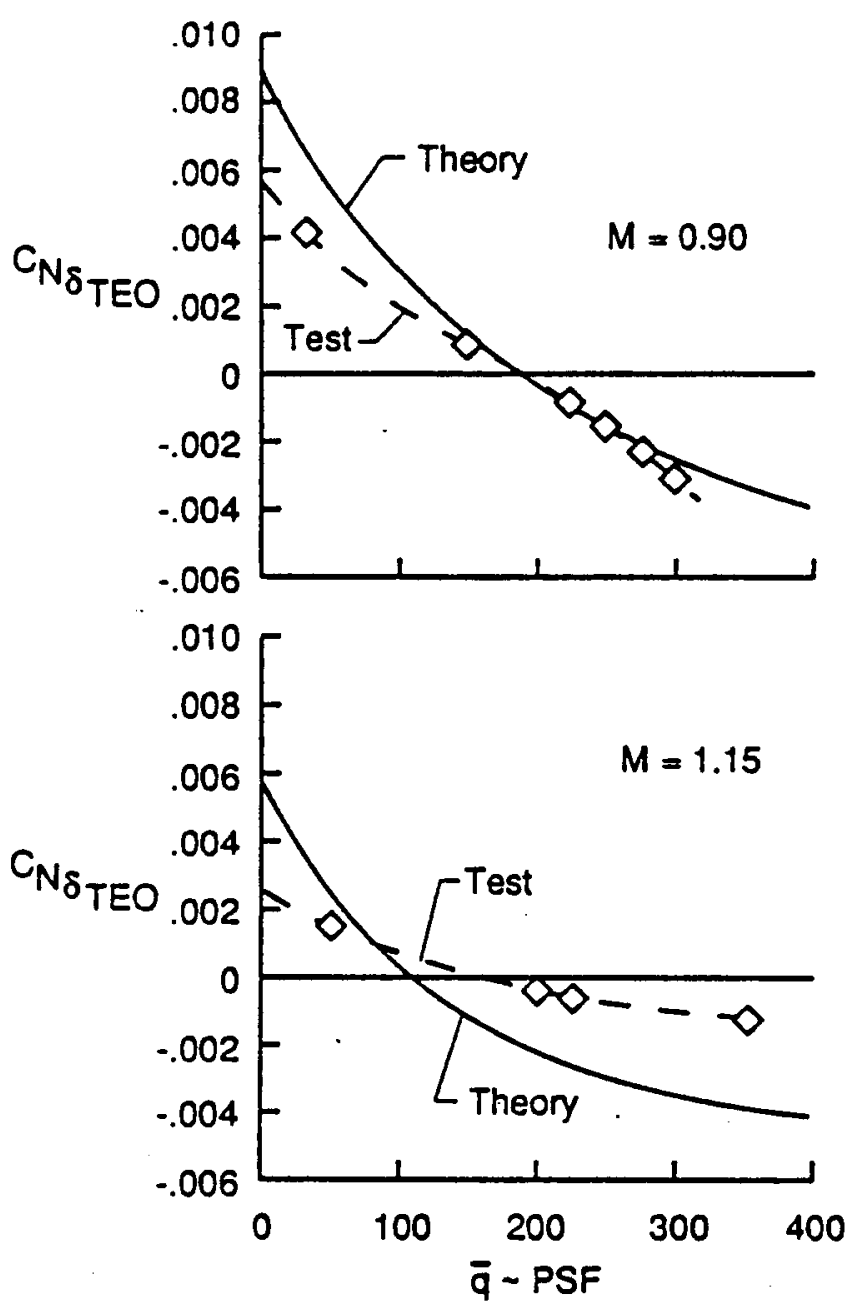

Figure 9 Trailing edge outboard control surface lift effectiveness with Mach number

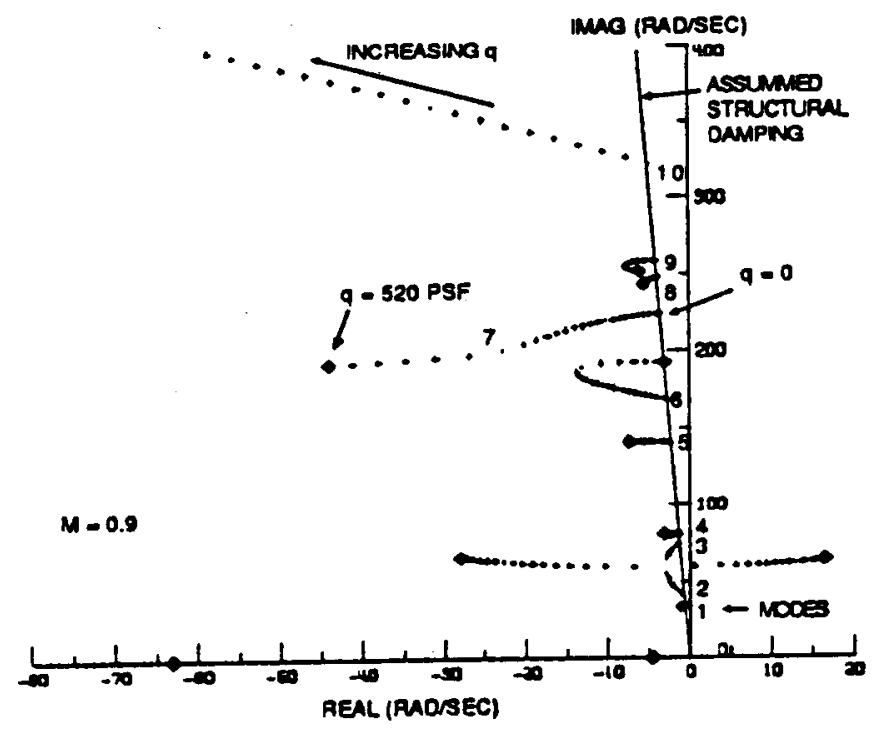

Figure 10 Dynamic pressure root locus plot for the symmetric modes, tip ballast "Stiff" model



Figure 11 Predicted symmetric and ancisymmetric flutter boundaries with Mach number

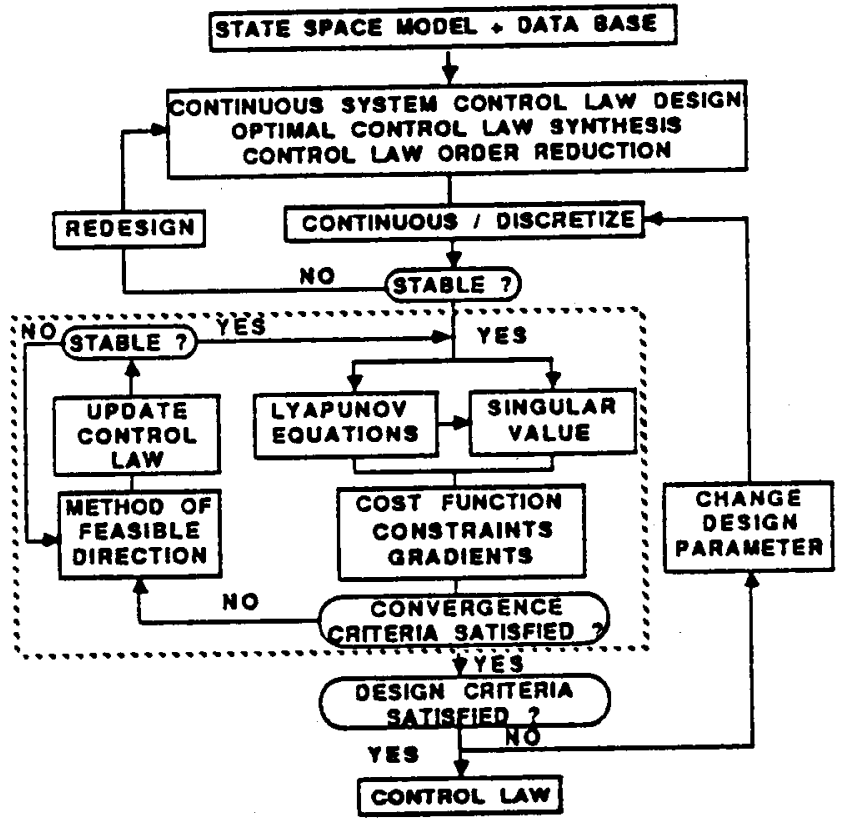

Figure 12 Schematic for an FSS synthesis procedure

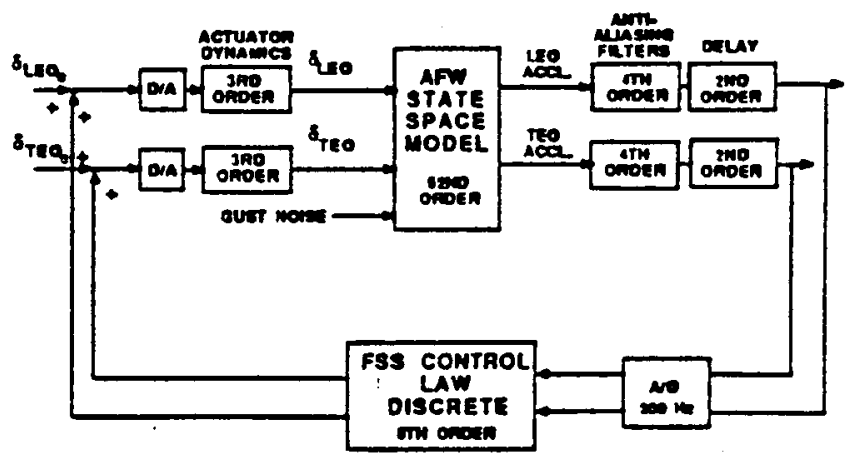

Figure 13 Block diagram of an FSS control system 
2.

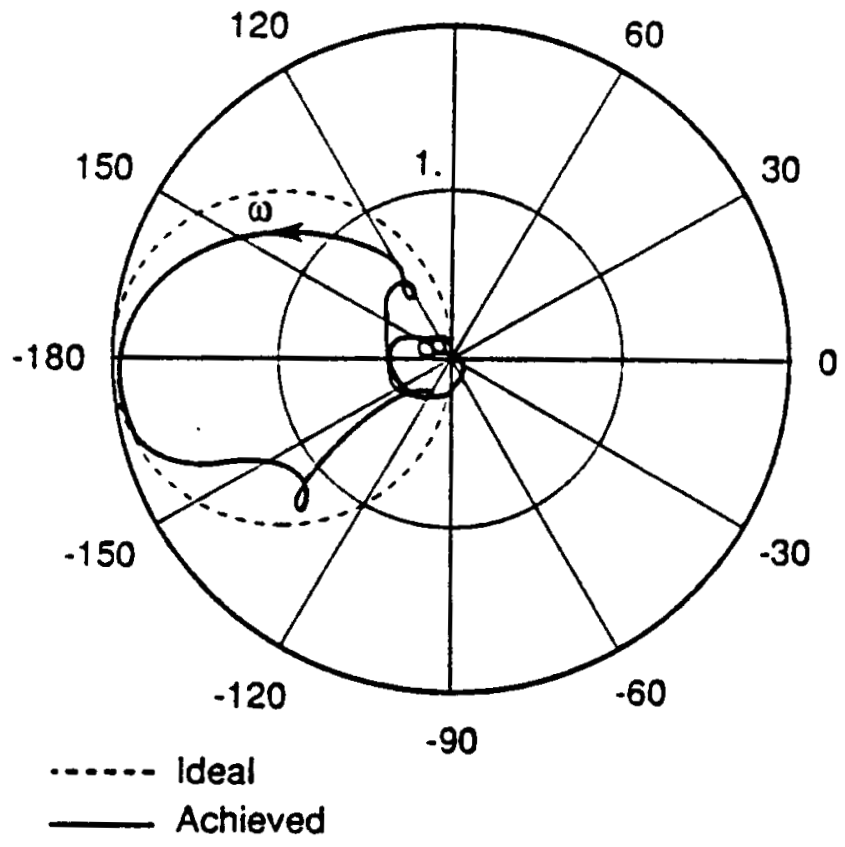

Figure 14 Predicted frequency response with sensor - blending

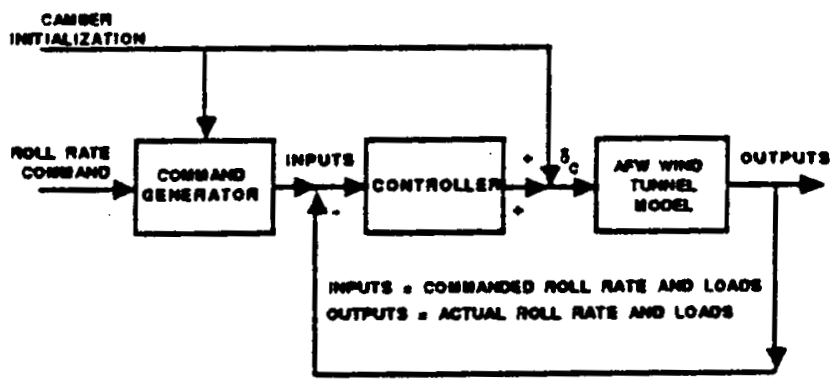

Figure 15 Block diagram of a RMLA control system

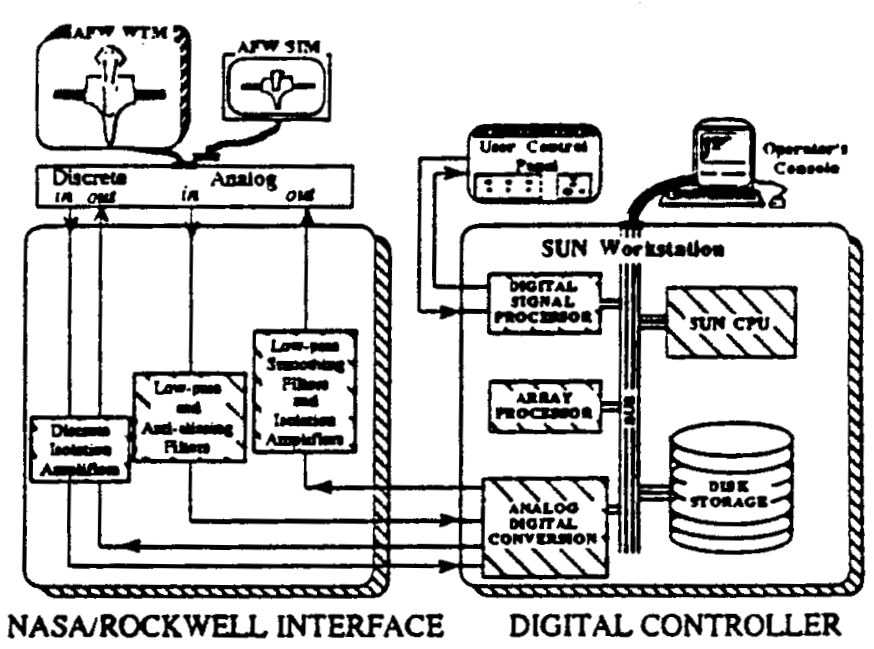

Figure 16 Schematic of the digital controller
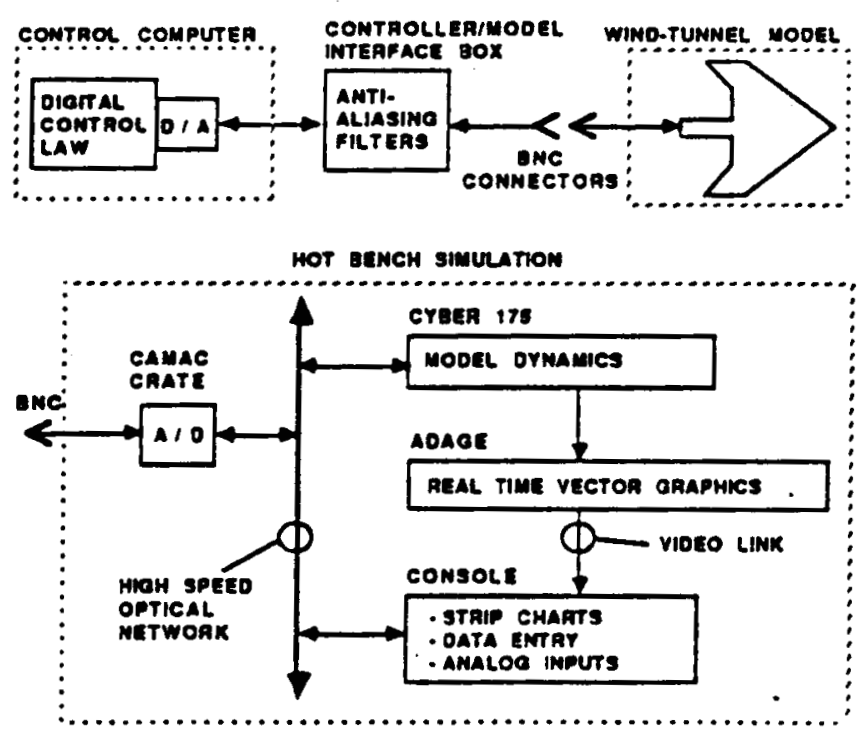

Figure 17 Schematic of the near-real-time simulator 


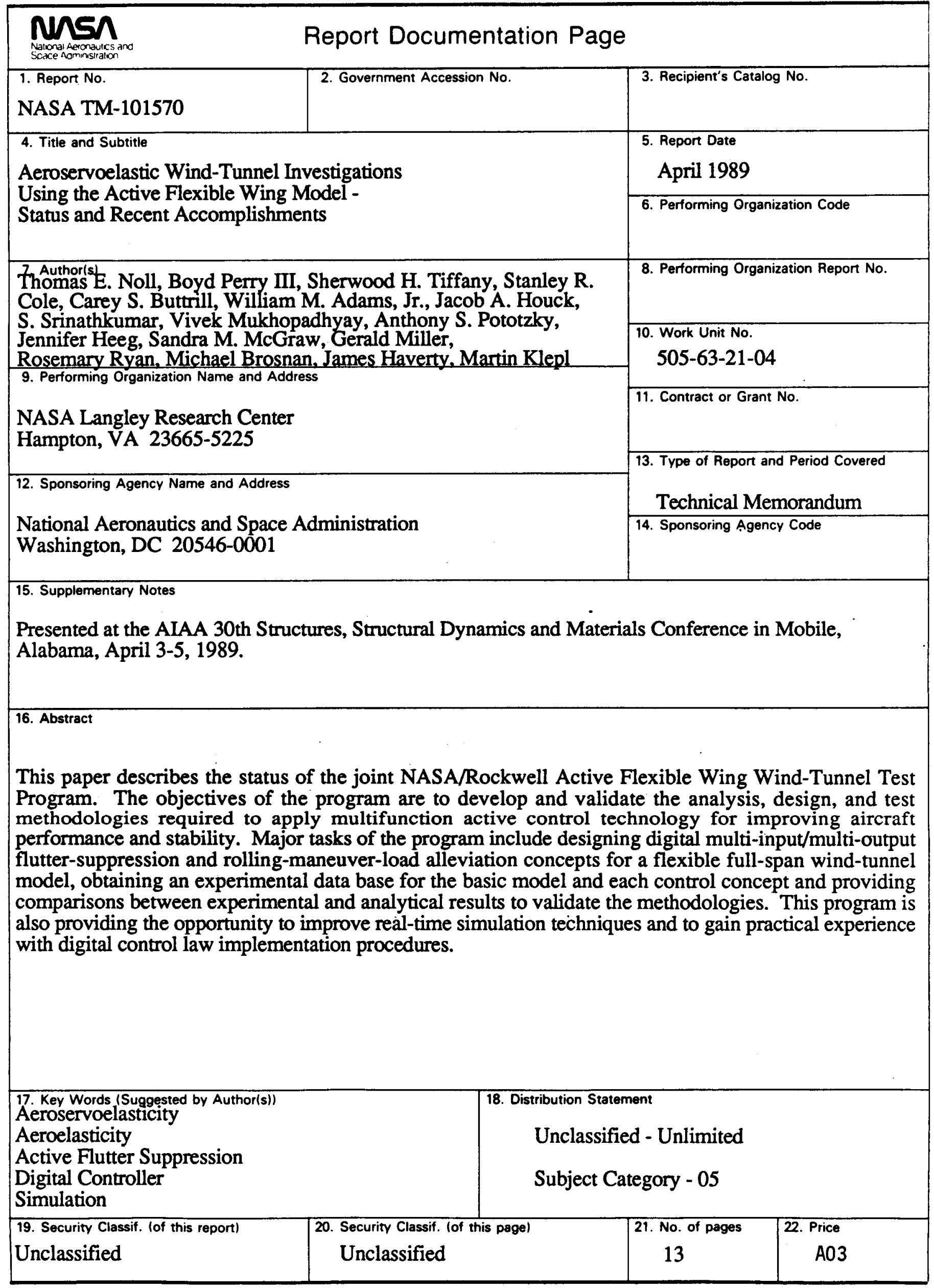

